\title{
Generation of solar spicules and subsequent atmospheric heating
}

\author{
CHEN YaJie*, SAMANTA Tanmoy \& TIAN Hui \\ School of Earth and Space Sciences, Peking University, Beijing 100871, China \\ Received January 20, 2020; accepted March 5, 2020; published online April 3, 2020
}

$\begin{array}{ll}\text { Citation: } & \begin{array}{l}\text { Chen Y J, Samanta T, Tian H. Generation of solar spicules and subsequent atmospheric heating. Sci China Tech Sci, 2020, 63: 2467-2468, https://doi. } \\ \text { org/10.1007/s11431-020-1555-6 }\end{array}\end{array}$

The temperature drops from $\sim 16$ million Kelvin at the core of the Sun to $5700 \mathrm{~K}$ at the solar surface (photosphere). However, the temperature increases with height above the photosphere, reaching one million degrees or more in the solar corona. Due to this high temperature, the corona naturally expands to the interplanetary space, forming the solar wind. What causes the temperature increase in the corona is one of the biggest mysteries in astronomy.

There are suggestions that small-scale dynamics in the chromosphere, such as ultraviolet bursts [1] and spicules [2], might play an important role in coronal heating. Spicules are magnetized jet-like plasma features with widths of $<500 \mathrm{~km}$. They dynamically shoot upward to heights of $\sim 5000 \mathrm{~km}$ above the photosphere. There are about one million spicules in the solar atmosphere at any given instant. These spicules may be the key to understanding coronal heating $[3,4]$.

Despite the importance of these spicules, the formation mechanisms of spicules are still under debate. There exist many hypotheses to explain the origin of spicules, such as the passage of shock waves [5], magnetic forces amplified through interactions between neutral and charged particles [6], warps in two-dimensional sheet-like structures [7], and "reconnection" between oppositely-directed magnetic fields [8]. However, direct observational evidence of the formation processes is still missing, mainly due to insufficient resolution and sensitivity.

Using high-spatial-resolution and high-time-cadence observations of the 1.6-m Goode Solar Telescope (GST) at the Big Bear Solar Observatory (BBSO), the world's largest-

*Corresponding author (email: chenyajie@pku.edu.cn) aperture solar telescope in operation, Samanta et al. [9] investigated the generation of many spicules and their possible contribution to coronal heating. GST observed numerous spicules at the high spatial resolution of $45 \mathrm{~km}$ and a high cadence of $3.5 \mathrm{~s}$ using the Hydrogen-alpha $(\mathrm{H} \alpha)$ spectral line and simultaneous photospheric magnetograms at a high spatial resolution of $150 \mathrm{~km}$ using the Fe I $1.56 \mu \mathrm{m}$ line. An overview of the observations is shown in Figure 1.

Their observations show that many spicules are associated with the interaction of magnetic fields with different polarities. These spicules mostly originate from the magnetic network, a grid-like pattern where the magnetic field is highly concentrated, and generally appear to be accompanied by the emergence of new magnetic elements with an opposite polarity to that of the existing magnetic network near their footpoints. Magnetic flux cancellation is identified at the footpoints of some spicules. These observations strongly suggest that magnetic reconnection between the strong network fields and the nearby weak fields drives many spicules.

In order to study the contribution of spicules to coronal heating, Samanta et al. [9] also examined possible coronal signatures of these spicules from simultaneous observations of the Atmospheric Imaging Assembly (AIA) onboard Solar Dynamics Observatory (SDO). They found obvious coronal signatures (visible in images of the AIA $171 \AA$ passband) at the top of most large spicules. Their on-disk quiet-Sun observations suggest that it is common for spicules to be heated to coronal temperatures. Samanta et al. [9] have provided the best evidence so far that magnetic reconnection in the lower solar atmosphere generates spicules and provides hot plasma flows into the corona, thus providing a direct link between 


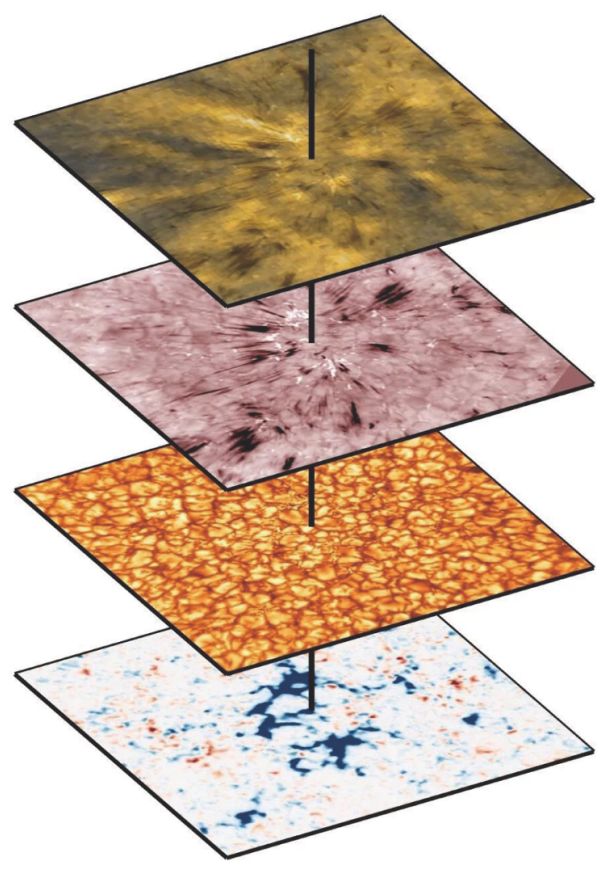

Figure 1 (Color online) Coordinated observations between GST and SDO. From bottom to top: photospheric magnetogram, images of the photosphere, chromosphere, and corona.

coronal heating and magnetic activities in the lower solar atmosphere.

In the next few years, the Advanced Space-based Solar Observatory (ASO-S), Solar Orbiter and Aditya-L1 missions will be launched, and the 4-m Daniel K. Inouye Solar Telescope (DKIST) will be in operation. These facilities will observe the solar atmosphere with high spatial and temporal resolutions using different passbands. Furthermore, the Parker Solar Probe (PSP) was successfully launched a year ago, and it can provide unique in-situ measurements of the corona and inner heliosphere [10]. Coordinated observations between these instruments, as well as theoretical investigations and advanced numerical simulations based on these observations, will help to understand the energy and mass transport through the solar atmosphere and coupling between different layers of the atmosphere.

1 Chen Y J, Tian H, Zhu X S, et al. Solar ultraviolet bursts in a coordinated observation of IRIS, Hinode and SDO. Sci China Tech Sci, 2019, 62: 1555-1564

2 Beckers J M. Solar spicules. Annu Rev Astron Astrophys, 1972, 10: $73-100$

3 De Pontieu B, McIntoshS W, Carlsson M, et al. The origins of hot plasma in the solar corona. Science, 2011, 331: 55-58

4 Tian H, DeLuca E E, Cranmer S R, et al. Prevalence of small-scale jets from the networks of the solar transition region and chromosphere. Science, 2014, 346: 1255711

5 Sterling A. Solar spicules: A review of recent models and targets for future observations. Sol Phys, 2000, 196: 79-111

6 Martínez-Sykora J, De Pontieu B, Hansteen V H, et al. On the generation of solar spicules and Alfvénic waves. Science, 2017, 356: 1269-1272

7 Judge P G, Tritschler A, Low B C. Thermal fine structure and magnetic fields in the solar atmosphere: Spicules and fibrils. Astrophys J, 2011, 730: L4

8 Ding J Y, Madjarska M S, Doyle J G, et al. Magnetic reconnection resulting from flux emergence: Implications for jet formation in the lower solar atmosphere? Astron Astrophys, 2011, 535: A95

9 Samanta T, Tian H, Yurchyshyn V, et al. Generation of solar spicules and subsequent atmospheric heating. Science, 2019, 366, 890-894

10 He J S, Tian H. Frontiers to be explored by the Parker Solar Probe Mission. Sci China Tech Sci, 2019, 62: 1481-1482 